\title{
EXTERNAL EQUIVALENCE CLASSES IN DECOMPOSITIONS OF SPACES
}

\author{
W. DEBSKI AND E. D. TYMCHATYN
}

\begin{abstract}
Let $X$ be an indecomposable continuum in the plane. Krasinkiewicz has shown that the union of the external composants of $X$ is a first category $F_{\sigma}$ set in $X$. We give extensions of this theorem to quite general decompositions of a space $X$ embedded in a locally connected ambient space $Y$.
\end{abstract}

1. Introduction. Consider the Cantor ternary set $X$ in the real line $E_{1}$. Let $X^{\prime}$ denote the set of points of $X$ which are not endpoints of any interval in $E_{1} \backslash X$. If $L$ is an arc in $E_{1}$ which meets $X^{\prime}$, then $L \cap X$ contains an open and, hence, a second category set in $X$.

Mazurkiewicz [5] proved that if $X$ is an indecomposable continuum in the plane, then the union of the accessible composants of $X$ is a first category $F_{\sigma}$-set in $X$. Krasinkiewicz [3] has given a far reaching generalization of Mazurkiewicz's theorem.

In this paper we will prove two theorems which will simultaneously generalize the above.

All spaces we consider are separable and metric. A continuum is a compact, connected metric space. If $(X, d)$ is a bounded metric space we let $C(X)$ denote the hyperspace of subcontinua of $X$ with the Hausdorff metric.

If $A \subset X$ we let $\mathrm{Cl}_{X}(A), \mathrm{Bd}_{X}(A)$, and $\operatorname{Int}_{X}(A)$ denote the closure, boundary, and interior, respectively, of $A$ in $X$.

Let $X$ be a subspace of a space $Y$ and let $R$ be an equivalence relation defined on $X$. For $x \in X$ we let $R(x)$ denote the $R$-equivalence class of $x$. For $A \subset X$ let $R(A)=\bigcup_{x \in A} R(x)$. An $R$-equivalence class $R(x)$ is said to be external in the sense of Krasinkiewicz if there exists a continuum $L$ in $Y$ such that $L \not \subset \mathrm{Cl}(X)$ and $R(x) \subset R(L \cap X) \neq X$. We let Ekras denote the union of the external (in the sense of Krasinkiewicz) $R$-equivalence classes of $X$.

An $R$-equivalence class $R(x)$ is said to be external in the sense of category if there exists a continuum $L$ in $Y$ such that $L \not \subset \mathrm{Cl}(X)$ and $R(x) \subset R(L \cap X)$ is of first category in $X$. We let Ecat denote the union of the external (in the sense of category) $R$-equivalence classes of $X$.

A continuum $X$ is indecomposable if it is not the union of two proper subcontinua. If $x \in X$, the composant of $x$ in $X$ is the union of all proper subcontinua of $X$ which contain $x$. It is well known that the composants of an indecomposable continuum $X$ are dense, pairwise disjoint, first category, $F_{\sigma}$-sets in $X$. Also, the equivalence relation $R$ on $X$ whose equivalence classes are the composants of $X$ has the property that $R=\bigcup_{i=1}^{\infty} R_{i}$ where each $R_{i}$ is closed in $X \times X$.

Received by the editors May 30, 1986.

1980 Mathematics Subject Classification (1985 Revision). Primary 54B15, 54E50; Secondary $54 \mathrm{~F} 20$.

Research was supported in part by NSERC grant A5616. 
Our first proposition shows that for the very important case of indecomposable continua in the 2 -sphere the two notions of externality coincide.

PROPOSITION 1. If $X$ is a compact space contained in a space $Y$ and $R=$ $\bigcup_{i=1}^{\infty} R_{i}$ is an equivalence relation on $X$ such that each $R_{i}$ is closed in $X \times X$ and each $R(x)$ is dense in $X$, then Ekras $=$ Ecat.

PROOF. Since $X$ is of second category it is clear that Ecat $\subset$ Ekras. If $R(z) \subset$ Ekras let $K$ be a continuum in $Y$ such that $K \not \subset \mathrm{Cl}_{Y}(X)$ and $R(z) \subset R(K \cap X) \neq X$. For each positive integer $i$ let

$$
L_{i}=\left\{y \in R(K \cap X) \mid(x, y) \in R_{i} \text { for some } x \in K\right\} .
$$

Then $L_{i}$ is closed in $X$ and $R(K \cap X)=\bigcup_{i=1}^{\infty} L_{i}$. Since $R(K \cap X) \neq X$ and each $R(x)$ is dense in $X$ it follows that $L_{i}$ is nowhere dense in $X$. Hence, $R(K \cap X)$ is of first category in $X$.

COROLlARY 2. Let $X$ be an indecomposable continuum contained in a space $Y$ and let $R$ be the decomposition of $X$ into composants. Then Ecat $=$ Ekras, i.e. the two definitions of external composants coincide.

Krasinkiewicz [3] proved the following remarkable extension of Mazurkiewicz's theorem:

THEOREM (KRASINKIEWICZ). If $X$ is an indecomposable continuum in the 2sphere $S^{2}$ and $R$ is the decomposition of $X$ into composants, then Ekras is a first category $F_{\sigma}$-set in $X$.

In [2] the authors extended Krasinkiewicz's theorem in several ways.

THEOREM (DEBSKI AND TYMCHATYN). Let $X \subset S^{2}$ be such that each nonempty open subset of $X$ is of second category. Let $R$ be an equivalence relation on $X$ such that each $R(x)$ is continuum connected, of first category in $X$, and dense in $X$. Then Ekras is a first category $F_{\sigma}$-set in $X$.

It is the purpose of this paper to prove analogues of some of the theorems in [2] concerning Ekras in $S^{2}$ for Ecat in general ambient spaces.

\section{Main results.}

THEOREM 3. Let $X$ be a subspace of a locally connected separable, metric space $Y$. Let $R$ be an equivalence relation on $X$ such that each $R(x)$ is continuum connected. Suppose $X^{\prime} \subset X$ such that $X \backslash X^{\prime}$ is of first category in $X$ and for each ordered pair $(U, V)$ of disjoint open sets in $Y$ with $U \cap X \neq \varnothing \neq V \cap X$ and for each $x \in X^{\prime} \cap V, Y \backslash U$ is separated by $R(x)$ between two points of $V \cap X$. Then Ecat is a first category set in $X$.

PROOF. Clearly, we may suppose $X$ is of second category or there is nothing to prove. Let $\mathcal{V}=\left\{V_{1}, V_{2}, \ldots\right\}$ be a basis of open connected sets for the topology of $Y$. Let $\mathcal{U}=\left\{U_{1}, U_{2}, \ldots\right\}$ be the elements of $\mathcal{V}$ which meet $X$. If $\mathrm{Cl}_{Y}\left(V_{i}\right) \cap$ $\left(\mathrm{Cl}_{Y}\left(X \cup U_{n}\right)\right)=\varnothing$ let

$$
D_{n, i}=\bigcup\left\{C \cap X \mid C \in C\left(Y \backslash U_{n}\right), C \cap \mathrm{Cl}_{Y}\left(V_{i}\right) \neq \varnothing\right.
$$
and $R(C \cap X)$ is of first category in $X\}$.

Otherwise, let $D_{n, i}=\varnothing$. 
Clearly, $D_{n, i} \subset$ Ecat. Now, let $R(x)$ be an external $R$-equivalence class of $X$. Let $L$ be a continuum in $Y$ which meets both $R(x)$ and $Y \backslash \mathrm{Cl}_{Y}(X)$ and such that $R(L \cap X)$ is of first category in $X$. Let $K$ be a continuum in $R(x)$ from $x$ to $L$. Then $R((K \cup L) \cap X)=R(L \cap X)$ so there exists $y \in X \backslash R(L \cap X)$. Let $U_{n}$ be a basic neighborhood of $y$ such that $\mathrm{Cl}_{Y}\left(U_{n}\right) \cap(L \cup K)=\varnothing$. Let $z \in L \backslash \mathrm{Cl}_{Y}(X)$ and let $V_{i}$ be a basic neighborhood of $z$ such that $\mathrm{Cl}_{Y}\left(V_{i}\right) \cap \mathrm{Cl}_{Y}\left(U_{n} \cup X\right)=\varnothing$. Then $x \in D_{n, i}$. So $\bigcup D_{n, i}=$ Ecat.

Let $n$ and $i$ be fixed integers. To prove Ecat is of first category in $X$ it suffices to prove that the interior in $X$ of $\mathrm{Cl}_{X}\left(D_{n, i}\right)$ is of first category in $X$. Just suppose $m$ is a positive integer such that $U_{m} \cap\left(V_{i} \cup U_{n}\right)=\varnothing$ and $U_{m} \cap X \subset \mathrm{Cl}_{X}\left(D_{n, i}\right)$. Let $L_{1}, L_{2}, \ldots$ be continua in $Y \backslash U_{n}$ such that for each $j L_{j} \cap \mathrm{Cl}_{Y}\left(V_{i}\right) \neq \varnothing, R\left(L_{j} \cap X\right)$ is of first category in $X$, and $U_{m} \cap X \subset \mathrm{Cl}_{X}\left(\bigcup_{j=1}^{\infty} L_{j} \cap X\right)$.

Let $x \in X^{\prime} \cap U_{m}$. Then $R(x)$ separates $Y \backslash U_{n}$ between two points of $X \cap U_{m}$. Since $V_{i}$ is connected, $R(x)$ separates $Y \backslash U_{n}$ between $V_{i}$ and a point of $X \cap U_{m}$. Since $Y$ is hereditarily normal, a closed subset of $R(x)$ separates $Y \backslash U_{n}$ between $V_{i}$ and a point of $X \cap U_{m}$. Since $\bigcup_{j=1}^{\infty} L_{j} \cap X$ is dense in $X \cap U_{m}$ and each $L_{j}$ meets $\mathrm{Cl}_{Y}\left(V_{i}\right)$ it follows that $R(x)$ meets some $L_{j}$.

Hence $X^{\prime} \cap U_{m} \subset \bigcup_{j=1}^{\infty} R\left(L_{j} \cap X\right)$. It follows that $U_{m}$ is of first category in $X$ since $R\left(L_{j} \cap X\right)$ and $X \backslash X^{\prime}$ are of first category in $X$. Since the interior in $X$ of $\mathrm{Cl}_{X}\left(D_{n, i}\right)$ is contained in the union of the countable family of sets $\left\{U_{j} \cap X \mid j=\right.$ $1,2, \ldots\}$ it follows that the interior in $X$ of $\mathrm{Cl}_{X}\left(D_{n, i}\right)$ is of first category in $X$.

We give an example to show that the condition in Theorem 3 that $R(x)$ is continuum connected is essential.

EXAMPLE 4. Let $K_{2}$ be Knaster's simplest indecomposable continuum,

$$
K_{2}=\lim _{\longleftarrow}\left(I_{i}, f_{i}^{j}\right)
$$

where each $I_{i}=[0,1]$ and $f_{i}^{i+1}=f$ is the mapping $f:[0,1] \rightarrow[0,1]$ given by

$$
f(x)= \begin{cases}2 x & \text { if } x \in\left[0, \frac{1}{2}\right], \\ 2-2 x & \text { if } x \in\left[\frac{1}{2}, 1\right] .\end{cases}
$$

Let $K_{2}$ have its usual embedding in the plane (see $[4$, p. 205]). Let $\underline{0}=(0,0,0, \ldots)$ be the endpoint of $K_{2}$. Let $F$ be the composant of $K_{2}$ which contains $\underline{0}$. Then each point of $F$ is accessible. Let

$$
F^{1}=F \backslash\left\{(x, x / 2, x / 4, \ldots) \mid 0 \leq x<\frac{1}{2}\right\} .
$$

Since $F \backslash F^{\mathbf{1}}$ has $c$ points and $K_{2}$ has $c$ composants let $g: F \backslash F^{\mathbf{1}} \rightarrow\{C \mid C$ is a composant of $\left.K_{2}\right\}$ be a one-to-one onto correspondence. Define an equivalence relation $R$ on $K_{2}$ by letting $R(x)=\{x\} \cup g(x)$ for each $x \in F \backslash F^{1}$ such that $g(x) \neq F$. If $g(x)=F$ let $R(x)=\left(F \backslash F^{1}\right) \cup\{x\}$. Each $R(x)$ is connected (but not continuum connected) since composants of $K_{2}$ and $F \backslash F^{1}$ are dense connected sets in $K_{2}$. Krasinkiewicz showed that each composant of $K_{2}$ different from $F$ separates $S^{2}$ locally in the required way. Each $R$-equivalence class of $K_{2}$ is accessible in the plane.

THEOREM 5. Let $X$ be a subspace of a compact, locally connected, metric space $Y$. Suppose $R$ is an equivalence relation on $X$ such that each $R(x)$ is continuum connected, dense in $X$, and of first category in $X$. Suppose $X^{\prime} \subset X$ such that 
$X \backslash X^{\prime}$ is of first category in $X$ and for each pair $(U, V)$ of open sets in $Y$ such that $U \cap X \neq \varnothing \neq V \cap X$ and for each $x \in X^{\prime}, R(x)$ separates $Y \backslash U$ between two points of $X \cap V$. Then Ecat is a first category $F_{\sigma}$-set in $X$.

ProOF. Let $\mathcal{V}, \mathcal{U}$, and $D_{n, i}$ be as in Theorem 3. Then Ecat $=\bigcup D_{n, i}$ as in Theorem 3. To prove the theorem it suffices to prove that each $D_{n, i}$ is closed in $X$.

Let $n$ and $i$ be fixed positive integers and let $\left\{x_{j}\right\}_{j=1}^{\infty}$ be a sequence in $D_{n, i}$ which converges to $x \in X$. For each positive integer $j$ let $L_{j}$ be a continuum in $Y \backslash U_{n}$ from $x_{j}$ to $\mathrm{Cl}_{Y}\left(V_{i}\right)$ and such that $R\left(L_{j} \cap X\right)$ is of first category in $X$. By passing to a subsequence if necessary we may suppose $\left\{L_{j}\right\}_{j=1}^{\infty}$ converges to a continuum $L$ in the compact metric space $C\left(Y \backslash U_{n}\right)$. Then $x \in L$ and $L \cap \mathrm{Cl}_{Y}\left(V_{i}\right) \neq \varnothing$.

If $R(L \cap X)$ is of first category in $X$ we are done. Suppose, therefore, that $R(L \cap X)$ is of second category in $X$. By Theorem 3 Ecat is of first category in $X$. Hence, $R(L \cap X) \backslash\left(\left(X \backslash X^{\prime}\right) \cup\right.$ Ecat $) \neq \varnothing$. Let $y \in R(L \cap X) \backslash\left(\left(X \backslash X^{\prime}\right) \cup\right.$ Ecat $)$. Since $R(y)$ is dense in $X$ we may suppose without loss of generality that $y \in U_{n}$. Let $\left\{U_{n_{j}}\right\}_{j=1}^{\infty}$ be a basis of neighborhoods of $y$ chosen from $\mathcal{U}$ and such that $U_{n_{j}} \subset U_{n}$ for each $j$.

For each $z \in R(L \cap X) \backslash R(y)$ and each $j$ let $A_{z, j}$ denote the union of the continua in $R(z) \backslash U_{n_{j}}$ which meet $L$. Let $Q_{j}=\bigcup\left\{A_{z, j} \mid z \in R(L \cap X) \backslash R(y)\right\}$. Since each $R(z)$ is continuum connected, $R(L \cap X)=\bigcup_{j=1}^{\infty} Q_{j} \cup R(y)$. Since $R(L \cap X)$ is of second category in $X$ and $R(y)$ is of first category in $X$ there exists an integer $r$ such that $Q_{r}$ is of second category in $X$. Hence, there exists an integer $m$ such that $Q_{r}$ is dense in $U_{m} \cap X$ and $U_{m} \subset \mathrm{Cl}_{Y}\left(U_{m}\right) \subset Y \backslash \mathrm{Cl}_{Y}\left(U_{n_{r}}\right)$.

Since $y \in X^{\prime}, R(y)$ separates $Y \backslash U_{n_{r}}$ between two points of $U_{m} \cap X$. Since $Y$ is hereditarily normal, a closed subset $A$ of $R(y)$ separates $Y \backslash U_{n_{r}}$ between two points of $U_{m} \cap X$. Since $Q_{r}$ is dense in $U_{m} \cap X, A$ separates $Y \backslash U_{n_{r}}$ between two points $a$ and $b$ of $Q_{r}$. By the definition of $A_{a, r}$ and $A_{b, r}$ there exist continua in $R(a)$ (resp. $R(b)$ ) from $a$ to $L$ (resp. from $b$ to $L$ ). These continua miss $A$. Hence, $A$ separates two points $a^{\prime}$ and $b^{\prime}$ of $L$ in $Y \backslash U_{n_{r}}$. Since the sequence $\left\{L_{j}\right\}_{j=1}^{\infty}$ converges to $L$ there is a positive integer $p$ such that $A$ separates two points of $L_{p}$ in $Y \backslash U_{n_{r}}$. Hence, $A \cap L_{p} \subset R(y) \cap L_{p} \neq \varnothing$. This is a contradiction since $R(y) \not \subset$ Ecat. The proof is complete.

\section{REFERENCES}

1. H. Cook, Subsets of indecomposable continua, Colloq. Math. 13 (1964), 37-43.

2. W. Debski and E. D. Tymchatyn, Composant-like decompositions of spaces (to appear).

3. J. Krasinkiewicz, On internal composants of indecomposable plane continua, Fund. Math. 84 (1974), 255-263.

4. K. Kuratowski, Topology. II, Academic Press, New York, 1968.

5. S. Mazurkiewicz, Sur les points accessibles des continues indécomposables, Fund. Math. 14 (1929), 107-115.

Department of Mathematics, University of SASKatchewan, Saskatoon, CANADA S7N 0W0 (Current address of E. D. Tymchatyn)

Current address (W. Debski): Institure of mathematics, Silesian University, Katowice, Poland 